\title{
A New Interpretation on the Non-Newtonian Properties of Particle Mass
}

\author{
Donald C. Chang \\ Hong Kong University of Science and Technology, Hong Kong, China \\ Email: bochang@ust.hk
}

How to cite this paper: Chang, D.C. (2018) A New Interpretation on the Non-Newtonian Properties of Particle Mass. Journal of Modern Physics, 9, 215-240.

https://doi.org/10.4236/jmp.2018.92015

Received: December 13, 2017

Accepted: January 19, 2018

Published: January 22, 2018

Copyright (C) 2018 by author and Scientific Research Publishing Inc. This work is licensed under the Creative Commons Attribution International License (CC BY 4.0).

http://creativecommons.org/licenses/by/4.0/

\begin{abstract}
It is well known that the mass of a particle has properties different from Newtonian mechanics. First, it is speed-dependent. Second, it is convertible to energy. These properties were generally thought to be derived from the principle of relativity (PR). We have conducted a careful examination of the historical records and found that the non-Newtonian properties of mass were derived not so much based on PR, but more based on Einstein's intuitive thinking that radiation and matters behave similarly. This gives us a hint: Since both photon and electron can behave as a particle as well as a wave, can such a wave nature account for the deviations from Newtonian mechanics? Thus, we have developed a wave model to describe the motion of a free particle with or without rest mass. We found that both the speed-dependence of mass and the mass-energy equivalence can indeed be derived based on the wave properties of a particle. This wave hypothesis has several advantages; it can naturally explain why particles can be created in the vacuum and why a particle cannot travel faster than the speed of light.
\end{abstract}

\section{Keywords}

Mass, Mass-Energy Equivalence, Wave-Particle Duality, Vacuum, Relativity

\section{Introduction}

In Newtonian mechanics, the mass of an object is thought to be a constant and does not change with its speed. With the development of particle accelerators, it was discovered that such an understanding is not totally correct. Although the rest mass $\left(m_{0}\right)$ of a particle is constant, its moving mass $(m)$ is not. In fact, the moving mass is found to increase with speed ( $v$ ) following the relation $m=\gamma m_{0}$, where $\gamma=\left(1-v^{2} / c^{2}\right)^{-1 / 2}$. Thus, as the speed of a particle increases toward the speed of light $(c)$, the behavior of the particle can no longer be described by 
Newtonian mechanics. Furthermore, unlike in Newtonian mechanics, we now know the mass $m$ and energy $E$ cannot be regarded as two independent concepts; they are related by the relation $E=m c^{2}$ [1] [2] [3] [4]. This new understanding of mass-energy equivalence was a great discovery in modern physics.

A major challenge is to explain the physical basis for the above discoveries. Historically, these non-Newtonian properties of mass were thought to be results due to the principle of relativity (PR) [5] [6]. But a careful examination of the literature indicates that such thinking is not totally correct. First, these concepts had been suggested by various scientists before the publication of the special theory of relativity (STR) [7] [8] [9] [10]. Second, although such concepts were actively promoted by Einstein, his arguments were based on special hypothetical situations (which he called "thought experiments") instead of based on first principles. Third, many of such thought experiments had nothing to do with PR; some might even violate the requirement of PR itself. (For details, see Section 2 of this paper). Finally, the postulate of PR requires the vacuum to be empty so that there is no fixed resting frame in our universe [5]. Such a requirement, however, appears to be in conflict with the Standard Model of cosmology today and the current thinking of the quantum field theories [11] [12] [13], both of which do not regard the vacuum as an empty space (For more details, see Discussions).

In this paper, we would like to explore if there can be other explanations on the physical basis of the non-Newtonian properties of mass. More specifically, we will examine whether these non-Newtonian properties of mass are due to the fact that the particle is an excitation wave of the vacuum. (The detailed analysis is given in Section 3.) Our investigation is motivated by the following considerations: In Newtonian mechanics, a particle is treated as a rigid pointed object. But in quantum mechanics, we know a particle behaves more like a wave packet. In fact, the Planck's relation, the de Broglie relation and Heisenberg's Uncertainty Principle can all be attributed to the wave nature of the particle [14]. Naturally, we expect that a wave packet will behave differently from a rigid pointed object (see Figure 1). Could this wave nature explain the non-Newtonian properties of

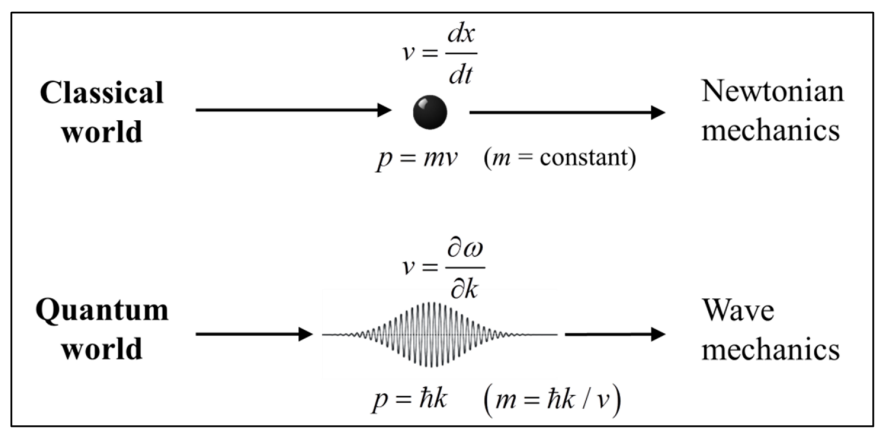

Figure 1. Different views of a particle. In classical world, a particle is regarded as a pointed mass. But in the quantum world, the particle is a wave packet. The velocity and mass of the particle are defined based on different physical concepts. So, it is not surprising that they can behave differently. (Here, $v$ is the particle velocity, $p$ is the momentum and $m$ is mass.) 
particle mass? This work is a report of our investigation on this question.

\section{A Brief Historical Review on the Derivation of Non-Newtonian Relations of Mass}

Before we introduce our new interpretation on the non-Newtonian properties of mass, let us review first their conventional interpretation. In many textbooks, the discovery of the non-Newtonian properties of mass is attributed to the prediction of STR. In the following, we will review the historical records and carefully trace the derivation of the two relations $E=m c^{2}$ and $m=m_{0}\left(1-v^{2} / c^{2}\right)^{-1 / 2}$. One may find that the history of their derivation was more complicated than what we were taught [15].

\subsection{In 1905, Einstein Proposed That Mass Can Be Speed-Dependent}

The first person to seriously suggest that mass is not a constant was A. Lorentz [10]. At the end of the $19^{\text {th }}$ century, Lorentz developed an aether theory to explain the results of Michelson-Morley experiment [16]. By defining mass as the ratio between force and acceleration, Lorentz's showed that the mass of an electron parallel to the direction of motion is $m_{L}=\gamma^{3} m_{0}$ and the mass perpendicular to the direction of motion is $m_{T}=\gamma m_{0}$, where $\gamma=1 / \sqrt{1-v^{2} / c^{2}}$ is the "Lorentz factor" [10].

In 1905, Einstein published his famous paper on relativity (we will refer this paper as his 1905a paper) [5]. He also realized that the mass of an object does not need to be constant. In this paper, he showed that the mass of an "electron" varies with its traveling speed in the following way:

$$
\left\{\begin{array}{l}
\text { Longitudinal mass }=\frac{m_{0}}{\left(1-v^{2} / c^{2}\right)^{3 / 2}} \\
\text { Transverse mass }=\frac{m_{0}}{1-v^{2} / c^{2}}
\end{array}\right.
$$

In order to obtain the above relations, Einstein explicitly stated that his definition of mass was relying on Newton's law, $F=m a$ [5]. So, like Lorentz, Einstein's definition of mass did not deviate much from that of Newton.

From the above, one can see that the longitudinal mass obtained by Einstein (Equation (1)) was the same as that of Lorentz; but Einstein's transverse mass (Equation (2)) was slightly different from Lorentz's result. As we know it today, the correct speed-dependence of the mass should be

$$
m=\frac{m_{0}}{\sqrt{1-v^{2} / c^{2}}},
$$

where $m$ is the moving mass and $m_{0}$ is the rest mass. This relation was first obtained by Lorentz and has been shown to agree with the results of a number of experiments [17] [18]. In modern textbooks of relativity, the speed-dependence of mass is described by Equation (3) rather than the original equations presented in Einstein's 1905a paper [19]. 


\subsection{In a Subsequent Paper, Einstein Proposed Emission of Radiation Is Related to Mass Loss}

Near the end of the $19^{\text {th }}$ century, many European physicists had become aware that the inertial mass can be related to the energy-content of an object [15]. For example, in 1881, J. J. Thomson studied the magnetic field generated by a moving charged sphere. He showed that the field could induce an effective mass on the sphere [7]. In 1889, Oliver Heaviside simplified Thomson's work and suggested that the effective mass should be $m=\frac{4}{3} \frac{E}{c^{2}}$ [20]. Later, Wilhelm Wien and Max Abraham got the same result, which became known as the "electromagnetic mass" [21] [22]. The relation of mass-energy equivalence $E=m c^{2}$ was first mentioned in a paper by Poincare in 1900 [9]. In 1904, Fritz Hasenöhrl wrote a series of papers entitled "On the theory of radiation in moving bodies", which also provided an elaborated study of the concept relating energy with mass [23] [24]. A detailed account of works regarding the mass-energy converting concept had been reviewed by W. Fadner [15], who showed that there were many discussions on this topic before 1905 .

Einstein clearly recognized the great importance of the mass-energy equivalence concept. In the same year after he published his 1905a paper on the principle of relativity, he published a very short paper entitled "Does the inertia of a body depend upon its energy-content?", which described his first derivation of the mass-energy equivalence relation [25]. This $1905 \mathrm{~b}$ paper was based on a "thought experiment" and its argument can be summarized as the following:

Consider a setup as illustrated in Figure 2. Let an object be placed at the origin of the stationary coordinate system $S$. A system moving along the $x$-axis with speed $v$ is designated as $S^{\prime}$. The total energy of the object as measured in the frame $S$ and $S^{\prime}$ are $E_{0}$ and $H_{0}$, respectively.

Now, let this object send out two identical pulses of light at opposite directions making an angle $\phi$ with $x$-axis. The energy of each of these light pulses is designated $1 / 2 L$ as measured in the $S$ frame. After this light pulse emission, the total energy of the object as measured in the frame $S$ and $S^{\prime}$ are denoted $E_{1}$ and

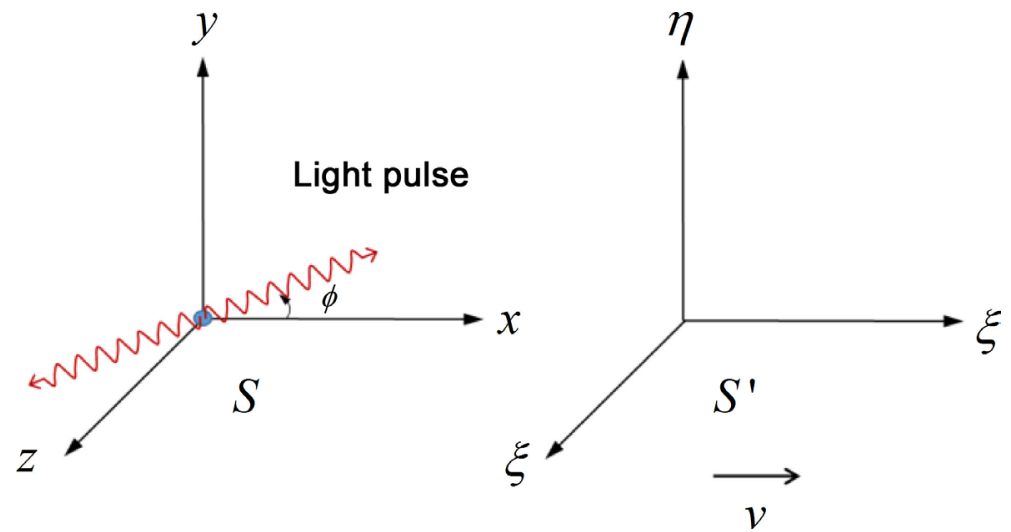

Figure 2. The setup to calculate mass-energy equivalence relation in Einstein's 1905b paper. 
$H_{1}$, respectively.

Einstein argued that, based on his theory of relativity, the energy of a light pulse measured in the stationary frame and the moving frame are different. Suppose the energy of the light pulse measured in the $S$ frame is $\ell$, then the energy of the light pulse in the $S^{\prime}$ frame will be

$$
\ell^{*}=\ell \frac{1-\frac{v}{c} \cos \phi}{\sqrt{1-v^{2} / c^{2}}} .
$$

Thus, in the above example, the energy of the opposite light pulse measured in the moving frame would become $\frac{1}{2} L \frac{1-\frac{v}{c} \cos \phi}{\sqrt{1-v^{2} / c^{2}}}$ and $\frac{1}{2} L \frac{1+\frac{v}{c} \cos \phi}{\sqrt{1-v^{2} / c^{2}}}$. Based on this argument, Einstein proposed that

$$
\left\{\begin{array}{l}
E_{0}=E_{1}+\frac{1}{2} L+\frac{1}{2} L \\
H_{0}=H_{1}+\frac{1}{2} L \frac{1-\frac{v}{c} \cos \phi}{\sqrt{1-v^{2} / c^{2}}}+\frac{1}{2} L \frac{1+\frac{v}{c} \cos \phi}{\sqrt{1-v^{2} / c^{2}}}=H_{1}+L \frac{1}{\sqrt{1-v^{2} / c^{2}}} .
\end{array}\right.
$$

By subtracting Equation (E-3) from Equation (E-2), one has

$$
H_{0}-E_{0}-\left(H_{1}-E_{1}\right)=L\left(\frac{1}{\sqrt{1-v^{2} / c^{2}}}-1\right) \text {. }
$$

Einstein then argued that, the difference between the energy measured in the $S$ and $S^{\prime}$ frames is mainly due to the kinetic energy $K$ of the object. That is,

$$
\left\{\begin{array}{l}
H_{0}-E_{0}=K_{0} \\
H_{1}-E_{1}=K_{1}
\end{array} .\right.
$$

Substitute Equation (E-5) into Equation (E-4), one has

$$
K_{0}-K_{1}=L\left(\frac{1}{\sqrt{1-v^{2} / c^{2}}}-1\right) \text {. }
$$

If $v \ll c$, one can use the Taylor expansion and ignore the higher order terms to get

$$
K_{0}-K_{1}=\frac{1}{2} \frac{L}{c^{2}} v^{2} .
$$

One may interpret the decrease in the kinetic energy as a loss of mass $(\Delta m)$ of the light-emitting object, i.e.,

$$
K_{0}-K_{1}=\frac{1}{2}(\Delta m) v^{2} .
$$

By comparing Equations ((E-7) and (E-8)), one may identify the mass loss as

$$
\Delta m=\frac{L}{c^{2}} .
$$


Einstein thus concluded: "If a body gives off the energy $L$ in the form of radiation, its mass diminishes by $L / C^{2}$." [25]

The derivation proposed by Einstein in the above thought experiment was very simple. However, it is not without problem. For example,

1) Equation (E-9) was not derived based on first principle. Instead, it is just a suggested result demonstrated from a hypothetical special situation.

2) The relation $E=m c^{2}$ was not an exact derivation; it was an approximation. It can only be applied when the speed $v$ is much smaller than $c$. (This criticism was raised by Planck [26]).

3) The argument for Equation (E-5) is not exactly correct. Strictly speaking, the energy of a particle is now known to be $E^{2}=c^{2} p^{2}+m_{0}^{2} c^{4}$. The total energy in general thus is not a linear sum of the resting energy and the kinetic energy.

4) This proposed "thought experiment" was not realistic. No one can perform such an experiment in reality. One cannot find a physical object that can spontaneously convert part of its mass to emit electro-magnetic radiation in opposite directions. If the light-emitting object is an elementary particle, the entire particle will decay (and it cannot emit photons in opposite directions). If the radiationemitting object is a group of atoms or molecules, the emission of photon(s) can only be due to the release of potential energy from their orbital electrons, not due to a decrease of kinetic energy caused by the loss of the object's rest mass, as suggested in this $1905 \mathrm{~b}$ paper.

\subsection{Einstein Subsequently Proposed More thought Experiments to Derive the Mass-Energy Relation}

Einstein was not satisfied with his derivation presented in the 1905b paper (as described above). He tried to use a different argument based on center of gravity. He proposed a new thought experiment in 1906 [27]. His argument is described in the book Special Relativity by A. P. French [19].

Suppose there is a rigid box with mass $M$ and length $L$ that is floating in space (see Figure 3). The box is initially stationary. At $t=0$, a burst of light wave (with radiation energy $E$ ) is emitted from the left end of the box. The radiation carries a momentum $E / c$. Since the total momentum of the system remains zero, the

(a)

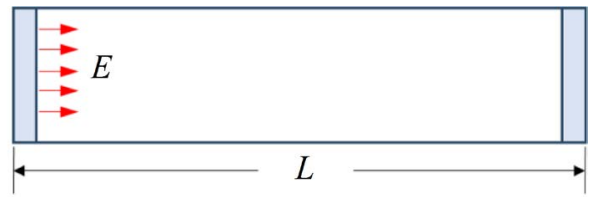

(b)

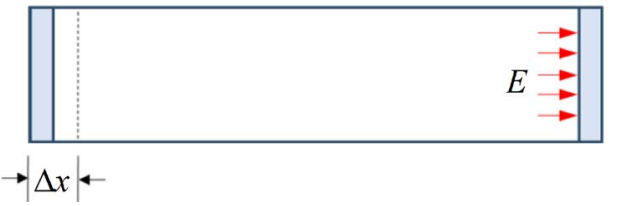

Figure 3. Einstein's thought experiment. A hypothetical experiment in which a box recoils from its initial position (a) to a final position (b) as a result of a burst of radiation energy traveling from one end of the box to the other. (Reproduced here based on [19]). 
box must acquire a momentum equal to $-E / c$. Hence the box will recoil with a speed $V$,

$$
v=-\frac{E}{M c} .
$$

At a later time, $t=\Delta t(\Delta t=L / c$, provided $v \ll c)$, the radiation wave reaches the right end of the box and its energy is totally absorbed. It conveys an impulse on the box equal and opposite to the initial burst of momentum. Therefore, it will bring the box to rest again. The net result of this process is to move the box through a distance $\Delta x$.

$$
\Delta x=v \Delta t=-\frac{E L}{M c^{2}} .
$$

Since this box is an isolated system, one can assume that the center of gravity of the box remains unchanged before and after the radiation process. In order to counter the movement of the rigid box, one must assume that the radiation should carry with it the equivalent of a mass $m$, such that

$$
m L+M \Delta x=0 .
$$

Combining Equations (5) and (6), we have

$$
m=\frac{E}{c^{2}} \text { or } E=m c^{2} .
$$

This suggests that the radiation wave can behave as a material object which has an effective mass equivalent to its energy divided by $c^{2}$.

One may notice that, although Einstein was able to partially justify the massenergy equivalence in this thought experiment, its derivation was not free of problems. For example,

- This thought experiment only demonstrated that a radiation wave could carry a mass that is proportional to its radiation energy. It did not show that an object with mass $m$ can convert its mass into an amount of energy $E=m c^{2}$.

- The argument in this thought experiment was not based on the principle of relativity. Instead, it was based on the assumption that the radiation should carry with it the equivalent of a mass $m$.

- As pointed out by Einstein himself in his later paper [28], this thought experiment violates the basic principle of STR, because it would assume information can be transmitted faster than the speed of light. Otherwise, the entire box cannot move simultaneously when a burst of radiation wave was emitted from its left end at $t=0$.

- It has been pointed out that this thought experiment was unrealistic and cannot be carried out in practice [29]. Even if it works, this thought experiment can only be regarded as a special case; it was not a general derivation of the mass-energy equivalence relation.

Following the 1905b and the 1906 papers, Einstein continued to publish several papers trying to give more convincing arguments about the derivation of the relation of mass-energy equivalence. His major works are summarized in 
Appendix A. Most of these works had been reviewed by various investigators in recent years [15] [20] [29] [30] [31]. As concluded in one of the reviews by Rothman: "(Einstein) was aware of the shortcomings of his derivation (in 1905 and 1906) and wrote a half dozen more papers over the next 40 years trying to patch things up but arguably never succeeded" [20]. A similar view was also given by Max Jammer in Chapter 3 of his book entitled "Concepts of mass in contemporary physics and philosophy" [30]. A number of physicists had expressed criticism on Einstein's derivation of the mass-energy equivalence relation [26] [30] [31] [32]. For example, Herbert Ives had criticized Einstein's 1905 derivation for using an argument of circular logic [26]. Ives' criticism was echoed by a number of investigators [30] [33]. On the other hand, other scientists had expressed disagreement with Ives [15] [34]. Some would even argue that "circularity is seldom a legitimate critique of scientific proposals" [15]. So, there was still controversy about Ives' criticism on Einstein's derivation.

A more elaborated review of Einstein's attempt to derive the relation of massenergy equivalence was recently given by Eugene Hecht, who published a series of papers on this subject in American Journal of Physics in the last few years [29] [35]. According to Hecht, "Einstein produced about 18 virtuoso derivations and demonstrations all aimed at establishing the mass-energy principle. We have shown that although each of them gave evidence for the applicability of $E_{0}=m c^{2}$ to a particular set of circumstances, no one derivation, or collection of them taken together, succeeded in providing a definitive proof of its complete generality". "The fact that Einstein continued to create demonstrations of the efficacy of $E_{0}=m c^{2}$ up to 1946 tells us that he knew the definitive proof had not been accomplished' [29].

\subsection{Einstein's Derivations Assumed That Matters Behave Like Radiation and Vice Versa}

As we pointed out in Section 2.1, Einstein's 1905 paper did not predict the correct speed-dependence of mass. But today, we are taught that STR gave the right prediction of $m=m_{0}\left(1-v^{2} / c^{2}\right)^{-1 / 2}$. What is the justification for that? After the correct formula for the speed-dependence of mass became known from experiments [17] [18] [36], people gave up Einstein's original argument in 1905 and used different ways to derive the speed-dependence of mass. As a demonstration of this new argument, let us briefly review the derivation of this $m=\gamma m_{0}$ relation in current standard textbooks.

A widely used textbook (which is part of The MIT Introductory Physics Series) is Special Relativity by A.P. French [19]. It bases its derivation of the relation $m=\gamma m_{0}$ on an assumption that matter behaves like radiation. For a photon, it can be shown that

$$
E=c p
$$

and

$$
m=\frac{E}{c^{2}} \text {. }
$$


From Equation (8), one can get

$$
E \mathrm{~d} E=c^{2} p \mathrm{~d} p .
$$

Although the above relations were derived for light, the author argued that these same relations are also valid for matter [19]. By integration of Equation (9), one can obtain,

$$
E^{2}=c^{2} p^{2}+E_{0}^{2},
$$

where $E_{0}^{2}$ is a constant of integration. From Newtonian mechanics, we know $p=m v$. By substituting this relation and Equation (7) into Equation (10), one can get

$$
m=\frac{m_{0}}{\sqrt{1-v^{2} / c^{2}}}
$$

where $m_{0}=E_{0} / c^{2}$.

The idea that radiation and matter behave similarly was a very interesting assumption. According to the Special Relativity textbook, this assumption was following the spirit of Einstein's work. Indeed, in many papers written by Einstein, he frequently implied that the energy involved in radiation and the mass of matter are conceptually convertible [25] [27] [37] [38]. One may say that, in Einstein's mind, mass is some sort of energy.

Hence, although Einstein had published many papers on the derivation of non-Newtonian relations of mass, most of his derivations were not based on the principle of relativity. Instead, his theoretical arguments were based on various hypothetical thought experiments which frequently implied that radiation and matter behave similarly (see Appendix A). Furthermore, some of these derivations were not free of flaws. One may conclude that, the general acceptance of these "non-Newtonian relations" was not based on the soundness of the theoretical argument. Instead, as pointed out by A. P. French, its real vindication is based on experimentally observed behavior of particles [19].

\section{The Non-Newtonian Properties of Mass Can Be the Results of Wave-Particle Duality}

From the above review, one can see that the key argument used by Einstein to derive the non-Newtonian relations of mass was less dependent on the principle of relativity, but more based on his intuitive thinking that matter and radiation behave similarly. In fact, such thinking had worked well for him. For example, it helped Einstein to formulate the correct relation to describe the photo-electric effect [39]. In such a study, the radiation wave was treated as a sort of matter (i.e., a pointed mass).

In this paper, we will try to follow the spirit of Einstein and see if we can find a better theoretical basis for the non-Newtonian properties of mass. Particularly, we wonder: Why does radiation and matter behave similarly? We think the answer could be: It is because at the quantum level, both radiation and matters are composed of waves! 
Based on this hint, we think the non-Newtonian properties of mass may be explained based on the phenomenon of wave-particle duality. In the following, we would like to explore if this is true.

\subsection{Both Radiation and Matter Wave Are Excitation Waves of the Vacuum}

The realization that matter and radiation can behave similarly is a great physical insight of many visionary physicists (including Lorentz, Poincare, Einstein and Planck) in early $20^{\text {th }}$ century. Their ideas were not only based on theories but also supported by experimental observations. It was discovered in a series of landmark experiments that photons (radiation wave) and electrons (particles making up matter) can have very similar physical properties [40] [41] [42] [43]. In fact, both photons and electrons were found to behave like particles as well as waves [40] [41] [42] [43]. This phenomenon was called "wave-particle duality". The fact that radiation wave (photon) can behave like a particle was demonstrated in Planck's study on black-body radiation and Einstein's study of photoelectric effect [39] [44]. On the other hand, massive particles (electrons) were also found to behave like waves. This was demonstrated by experiments in 1927 that electrons can be diffracted by a nickel crystal following the Bragg's law [43]. Using this wave property of electrons, people started to develop electron microscopes [45]. Apparently, the behavior of electron is very similar to photon.

In summary, it was well established in early $20^{\text {th }}$ century that:

1) The photon is a quantized radiation wave, but it can behave like a particle.

2) The electron is a particle with mass, but it can behave like a wave.

3) The electron and the photon behave very similarly in diffraction experiments.

From these observations, one may ask: Can the physical nature of both particles (photons and electrons) be similar? In the case of photon, we know it is an excitation wave of the vacuum; it is clearly a physical wave. In the case of the electron, it is not clear what its physical nature is. In the conventional teaching of quantum mechanics, the wave function for an electron is commonly interpreted as a probability function of finding the particle. This is generally referred to as the "Copenhagen interpretation" [46]. But as pointed out in our recent work, this interpretation does not preclude the possibility that the electron is a real physical wave packet [47]. In fact, if we assume the particle is a physical wave instead of a pointed object, it will be very easy to explain the experimental observations of electron diffraction by crystal. This physical wave model can also allow us to explain the observation that an electron can pass a double-slit and form an interference pattern [48]. Furthermore, this wave model can easily explain the creation and annihilation of particles. We know particles (including photons and electrons) can be created in the vacuum, or annihilated in the vacuum to give other particles [6]. If particles are not excitation waves of the vacuum, it will be very difficult to explain why something can be created from nowhere, or something can disappear into nowhere. 
Thus, it is reasonable to assume that both photons and electrons are excitation waves of the vacuum. In fact, we have shown recently that some of the quantum mechanical properties of a particle, including the Planck's relation, $E=h v$, de Broglie relation $p=\hbar k$, and Heisenberg's Uncertainty principle, can all be derived based on the assumption that a particle is a wave packet [14]. Based on the above considerations, we can formally hypothesize that [47]

1) Like the photon, a free particle (such as an electron) is an excitation wave of the vacuum.

2) Different types of free particles are different excitation modes of the vacuum.

Now, if a particle is a wave, naturally it would not behave like a bulk object as described in the Newtonian mechanics. This explains why a particle has nonclassical properties of mass. In the following, we will show that this wave hypothesis can naturally lead to the "non-Newtonian relations" of mass, including $E=m c^{2}$ and $m=m_{0}\left(1-v^{2} / c^{2}\right)^{-1 / 2}$.

\subsection{Mass-Energy Relationships in Matter Wave}

The idea that a massive particle behave like a wave was actively considered in the early days of quantum mechanics. This wave was called "matter wave", which is analogous to the "radiation wave" of a photon [46]. In our model, both radiation waves and matter waves are assumed to be excitation waves of the same vacuum. According to the Maxwell theory, the vacuum behaves like a dielectric medium [49] [50] [51]. If both radiation wave and matter wave are excitation waves of the same medium, they should obey the same wave equation, which is determined solely based on the physical properties of the vacuum. At this point, we already know the wave equation of a photon, which is an oscillating wave of the electro-magnetic field. The photon wave function can be described by the vector potential $A^{\mu}=(\phi, \boldsymbol{A})$. Using Maxwell equations, one can derive

$$
\nabla^{2} A^{\mu}-\frac{1}{c^{2}} \frac{\partial^{2} A^{\mu}}{\partial t^{2}}=0,
$$

where $c=1 / \sqrt{\mu_{0} \varepsilon_{0}}$ is the speed of light. ( $\varepsilon_{0}$ is permittivity and $\mu_{0}$ is permeability in free space). This may provide a very useful hint. Since both matter waves and radiation waves are thought to be excitation waves of the same vacuum medium, their wave equation should be similar. That means that the wave function of the matter wave $\psi$ may obey a wave equation that is similar to Equation (11). Indeed, in our recent model which treated the vacuum as a dielectric medium, we found that its wave motion is analogous to the oscillation in an elastic solid [47]. Using the technique of Helmholtz decomposition [52], one can demonstrate that the wave oscillation in the vacuum medium can be described by the following equation [47]:

$$
\nabla^{2} \psi-\frac{1}{c^{2}} \frac{\partial^{2} \psi}{\partial t^{2}}=0 .
$$

We propose that this is the basic wave equation (BWE) which is satisfied by all 
types of excitation waves in the vacuum. Different solutions of $\psi$ just represent different types of free particle in the physical world [47].

One can easily see that the simplest solution for BWE (i.e., Equation (12)) is a plane wave

$$
\psi_{\boldsymbol{k}} \sim \mathrm{e}^{i(\boldsymbol{k} \cdot \boldsymbol{x}-\omega t)},
$$

where $\boldsymbol{k}$ is the wave vector and $\omega$ is the frequency, and

$$
\omega=c k \text {. }
$$

We propose that this plane wave solution represents a radiation wave (a photon) which is a particle without rest mass. (Indeed, this plane wave is commonly used as the wave function of light in electrodynamics). The reason for this proposal is that, using the Planck's relation and the de Broglie relation, Equation (13A) becomes

$$
E=c p
$$

which is the known energy-momentum relation for a photon. For a particle with non-zero rest mass, the plane wave solution apparently is not a proper solution of BWE. This is not only because it fails to give the correct energy-momentum relation, it also does not meet our expectation for the behavior of a massive particle. We know a massive particle (such as an electron) behaves like a pointed mass in the classical limit. Hence, the probability of finding the particle should be somewhat localized to its trajectory. This means that its wave function not only depends on the spatial coordinate along its trajectory (i.e., $\hat{\boldsymbol{k}} \cdot \boldsymbol{x})$, its wave function should also vary in the transverse plane $(\hat{\boldsymbol{k}} \times \boldsymbol{x})$. Thus, a solution representing a massive particle cannot be a plane wave.

Indeed, the general solution of Equation (12) should vary with the spatial coordinates both in the longitudinal and transverse directions. For simplicity, one may assume such a wave function can be written as a product of the longitudinal component of the wave function $\left(\psi_{L}\right)$ and the transverse component of the wave function $\left(\psi_{T}\right)$, i.e.,

$$
\psi_{\boldsymbol{k}}(\boldsymbol{x}, t)=\psi_{T}(\hat{\boldsymbol{k}} \times \boldsymbol{x}) \psi_{L}(\hat{\boldsymbol{k}} \cdot \boldsymbol{x}, t),
$$

where $\psi_{L}$ describes the travelling wave along the particle's trajectory, and $\psi_{T}$ describes the wave function in the transverse plane. Substituting Equation (14) into Equation (12), and using the technique of separation of variables, one can obtain two coupled equations:

$$
\left\{\begin{array}{l}
{\left[\nabla^{2}-\frac{1}{c^{2}} \frac{\partial^{2}}{\partial t^{2}}\right] \psi_{L}(\hat{\boldsymbol{k}} \cdot \boldsymbol{x}, t)=\ell^{2} \psi_{L}(\hat{\boldsymbol{k}} \cdot \boldsymbol{x}, t)} \\
\nabla^{2} \psi_{T}(\hat{\boldsymbol{k}} \times \boldsymbol{x})=-\ell^{2} \psi_{T}(\hat{\boldsymbol{k}} \times \boldsymbol{x})
\end{array}\right.
$$

where $\ell$ is a fitting parameter. To simplify the solution of the above equations, let us denote the direction of the trajectory (i.e., $\hat{\boldsymbol{k}}$ ) as the $z$-axis. Equations $(15 \mathrm{~A})$ and $(15 \mathrm{~B})$ then can be solved easily to give 


$$
\begin{gathered}
\psi_{L}(\boldsymbol{x}, t) \sim \mathrm{e}^{i(k z-\omega t)} \\
\text { and } \psi_{T}(\boldsymbol{x}, t) \sim J_{n}(\ell r) \mathrm{e}^{ \pm i n \theta},
\end{gathered}
$$

where $J_{n}$ is Bessel function of the first kind; $n$ can be an integer or half integer; the coordinates $r$ and $\theta$ represent the radial distance and the azimuthal angle of the space vector in the transverse plane. The general solution of $\psi_{\boldsymbol{k}}(\boldsymbol{x}, t)$ thus is

$$
\psi_{\boldsymbol{k}}(\boldsymbol{x}, t)=a J_{n}(\ell r) \mathrm{e}^{ \pm i n \theta} \mathrm{e}^{i(k z-\omega t)},
$$

where $a$ is a normalizing constant. In order to satisfy the BWE, the wave parameters $\omega, \boldsymbol{k}$, and $\ell$ in Equation (16) are subjected to the condition

$$
\omega^{2}=\left(k^{2}+\ell^{2}\right) c^{2} .
$$

(This is called the "dispersion relation"). From Equation (16), one may see that the wave function representing a massive particle does not behave like a plane wave; instead, it behaves more like a vortex. Furthermore, the wave function not only oscillates in the longitudinal direction, it also oscillates in the transverse direction (with a wavelength proportional to $1 / \ell$ ). The question now is: From the dispersion relation shown in Equation (17), can one obtain the correct energy-momentum relation for a massive particle?

Using the quantum correspondence rules $E \rightarrow i \hbar \partial / \partial t$ and $\boldsymbol{p} \rightarrow-i \hbar \nabla$, one can show that $\omega$ and $\boldsymbol{k}$ are related to the energy $(E)$ and momentum $(p)$ of the particle, i.e.,

$$
\begin{array}{r}
E=\int \psi_{\boldsymbol{k}} * i \hbar \frac{\partial}{\partial t} \psi_{\boldsymbol{k}} \mathrm{d}^{3} x=\hbar \omega, \\
\text { and } \boldsymbol{p}=\int \psi_{\boldsymbol{k}} * \frac{\hbar}{i} \nabla \psi_{\boldsymbol{k}} \mathrm{d}^{3} x=\hbar \boldsymbol{k} .
\end{array}
$$

( $\hbar$ is Planck's constant divided by $2 \pi$ ). This reaffirms that, like the photon, a free particle with rest mass also obeys the Planck's relation and the de Broglie relation.

From the wave parameters in $\psi_{\boldsymbol{k}}$, we can also obtain the particle mass. Equation (17) can be rewritten as

$$
(\hbar \omega)^{2}=c^{2}\left(\hbar^{2} k^{2}+\hbar^{2} \ell^{2}\right) .
$$

Using the Planck's relation and the de Broglie relation, the above equation becomes

$$
\begin{aligned}
E^{2} & =c^{2} p^{2}+c^{2} \hbar^{2} \ell^{2}, \\
\text { or, } E & =\sqrt{c^{2} p^{2}+c^{2} \hbar^{2} \ell^{2}} .
\end{aligned}
$$

Since the particle is represented by a wave packet, the particle velocity $(v)$ is determined by its group velocity $v=\frac{\partial \omega}{\partial k}=\frac{\partial E}{\partial p} \quad$ [46]. Also, from classical mechanics, we know the particle mass $m$ is defined by its momentum $p=m v$. Using these two relations, one can solve Equation (20A) and obtain 


$$
m=\frac{\hbar \ell / c}{\left(1-v^{2} / c^{2}\right)^{1 / 2}} .
$$

We know at $v=0, m$ equals the rest mass $m_{0}$. Equation (21) then implies

$$
m_{0}=\frac{\hbar \ell}{c} \text {. }
$$

Combine Equations (21) and (22), we can see that the mass of a particle is indeed speed-dependent

$$
m=\frac{m_{0}}{\sqrt{1-v^{2} / c^{2}}} .
$$

Substituting Equation (22) into Equation (20), we can easily see that the dispersion relation for a free particle now leads to

$$
E^{2}=p^{2} c^{2}+m_{0}^{2} c^{4} \text {. }
$$

Furthermore, by combining the above two equations with $p=m v$, one can obtain

$$
E=m c^{2}
$$

Thus, for a particle with nonzero rest mass, it obeys the same mass-energy relation as that of a photon. At this point, we can easily see that, the photon is no different from other particles with nonzero rest mass. Both the photon and the massive particle are excitation waves of the vacuum. From Equation (23), one can see that, at $v \rightarrow 0$ (i.e., $\boldsymbol{p} \rightarrow 0$ ), the resting energy of the particle becomes

$$
E_{0}=m_{0} c^{2} .
$$

Thus, the relation between energy and momentum for a simple particle can also be written as

$$
E^{2}=p^{2} c^{2}+E_{0}^{2}
$$

This equation is the well-known energy-momentum relation of a particle with nonzero rest mass.

\subsection{Why a Photon Always Travels at a Constant Speed $c$ ? Why Must a Massive Particle Travel at a Speed Less than the Speed of Light?}

One advantage of this wave interpretation is that it can provide a clear physical basis to explain why light must travel at a constant speed of $c$ and no particle can travel faster than $c$. In classical mechanics, the vacuum is just an empty space; a particle is a rigid object. In principle, a particle should be able to travel at any speed. Then, how can one explain the observation that a particle cannot travel faster than the speed of light. What is the physical reason for that?

The wave model presented here can provide a simple answer to this question. According to our model, the particle is an excitation wave of the vacuum; its traveling speed is determined by the group velocity of the wave packet, which is generally slower than its phase velocity. This means that the maximum speed for 
a particle is its phase velocity. In our basic wave equation (i.e., Equation (12)), the phase velocity for all excitation waves in the vacuum is $c=1 / \sqrt{\mu_{0} \varepsilon_{0}}$. Hence, $c$ is entirely determined by the physical properties of the vacuum. (In another word, the second postulate of STR is really based on the physical form of the wave equation.)

Based on this wave model, it is easy to see that a photon should always travel at a constant speed of $c$. From the last section, we know the energy-momentum relation for a particle (i.e., Equation (10)) is related to the dispersion relation of the excitation wave, i.e.,

$$
\omega^{2}=\left(k^{2}+\ell^{2}\right) c^{2} .
$$

From this dispersion relation, one can directly calculate the traveling speed of the particle, which is equal to the group velocity of the wave packet, i.e., $\quad v=\frac{\partial \omega}{\partial k}$. For a photon, it has no rest mass, i.e., $\ell=0$; its dispersion relation is

$$
\omega=c k \text {. }
$$

Then,

$$
v=\frac{\partial \omega}{\partial k}=c .
$$

Its group velocity is the same as its phase velocity $(\omega / k=c)$. This explains why a photon always travels at a constant speed $c$.

For a massive particle, $\ell \neq 0$. From Equation (17), its group velocity is

$$
v=\frac{\partial \omega}{\partial k}=\frac{c k}{\sqrt{k^{2}+\ell^{2}}}<c .
$$

This explains why the speed of a massive particle is not constant and its maximum speed cannot exceed the speed of light, $c$. Furthermore, the fact that the maximum speeds for all particles (with or without rest mass) are the same suggests that massive particles and photons are excitation waves of the same medium (i.e., the vacuum).

\section{Discussions}

There are several advantages with the model proposed in this work. First, it is conceptually simple. Second, this model is based on wave-particle duality; it has a well-established foundation in quantum physics. Third, from the above analysis, one can see that our wave hypothesis can provide a simple physical basis to justify Einstein's second postulate in STR. This model suggests a new interpretation on the non-Newtonian properties of mass. In the following, we would like to further examine if this new interpretation is consistent with current physical theories.

\subsection{Is the New Interpretation Consistent with the Requirement of Lorentz Invariance?}

Historically, the principle of relativity has been incorporated into the mainstream 
of modern physics, particularly in quantum field theory (QFT). It is usually assumed that the equations of motion in QFT remain unchanged under the Lorentz transformation. (This is called "Lorentz covariant" or "Lorentz invariant"). Then, is our model consistent with this practice?

The answer is "yes". As one can see from Equation (12), the basic wave equation used in our model is indeed Lorentz invariant. The form of this equation will not change when the coordinate system is converted from $(t, x, y, z)$ to $\left(t^{\prime}, x^{\prime}, y^{\prime}, z^{\prime}\right)$ using the Lorentz transformation.

Furthermore, since our basic wave equation is Lorentz invariant, a state vector can be represented by the Minkowski 4-vector. For example, the spacetime can be written as $\boldsymbol{x}^{\mu}=\left(x^{0}, x^{1}, x^{2}, x^{3}\right)=(c t, x, y, z)=(c t, \boldsymbol{x})$. This is consistent with the common practice in the conventional QFT, where most state vectors are represented using the notations of 4-vectors. Also, since our model is fully consistent with the Maxwell theory, one can also write the charge-current densities as $\boldsymbol{J}^{\mu}=(\rho, \boldsymbol{J})$ and the potentials as $\boldsymbol{A}^{\mu}=(\phi, \boldsymbol{A})$.

Finally, since we showed in this model that the energy and momentum of a particle satisfied Equation (10),

$$
E^{2}=p^{2} c^{2}+E_{0}^{2}
$$

it can be rewritten as

$$
E^{2}-p^{2} c^{2}=E_{0}^{2} .
$$

One can then express the energy-momentum 4-vector as $\boldsymbol{p}^{\mu}=(E, \boldsymbol{p})$ and require its dot product to be a scalar. (Note: In some of the 4-vector notations, it is assumed $c=1$ ). This agrees with the common practice in QFT.

\subsection{Is This Wave Model Consistent with the Wave Equations Currently Used in Quantum Mechanics?}

In this work, we suggested that both radiation wave and matter wave are excitation waves of the vacuum. Their motion can be described by a wave equation which appears in form as a 4-dimensional Laplace equation (i.e., Equation (12)). We call this equation the "Basic Wave Equation" (BWE). Different solutions of the BWE would represent different particles. One may ask: Is this wave model consistent with the known wave equations used in quantum mechanics today?

In the conventional theories, different particles are thought to satisfy different quantum wave equations. For example, the photon is supposed to satisfy the wave equation of light, the scalar particle is supposed to satisfy the Klein-Gordon equation, and the electrons are supposed to satisfy the Dirac equation (or the Schrödinger equation). In order to reconcile our wave model with the conventional quantum theories, one must demonstrate that all conventional quantum equations for different particles are derivable from the BWE. For particles without mass (i.e., radiation waves), it is easy to see that our BWE can directly lead to the wave equation of light (i.e., Equation (11)). But for particles with rest mass (i.e., matter waves), can the BWE be consistent with their quantum wave equations? 
For example, can one derive the Klein-Gordon equation or the Dirac equation from the BWE?

This indeed can be done. Recall that the general solution of BWE contains both a longitudinal component $\left(\psi_{L}\right)$ and a transverse component $\left(\psi_{T}\right)$. The wave function describing the movement of a particle along its trajectory is basically represented by the longitudinal wave equation $\left(\psi_{L}\right)$, which satisfies Equation (15A). Since we now know $\ell$ is connected with the rest mass $m_{0}$ through Equation (22), Equation (15A) becomes

$$
\left[\nabla^{2}-\frac{1}{c^{2}} \frac{\partial^{2}}{\partial t^{2}}\right] \psi_{L}-\left(\frac{m_{0} c}{\hbar}\right)^{2} \psi_{L}=0 .
$$

This is identical to the Klein-Gordon equation. Thus, there is a natural consistency between the BWE and the Klein-Gordon equation. The only assumption here is that the wave function of the Klein-Gordon equation describes only the movement of a free particle along its trajectory.

From the Klein-Gordon equation, one can further derive the Dirac equation by linearizing Equation (27). This requires treating the wave function as a spinor and the wave equation must be written in the matrix form [47] [53]. This procedure has been worked out by Dirac [53]. For details, one can see our previous publication [47].

Once the Dirac equation is derived, one can further demonstrate that the Schrödinger equation can be derived based on the Dirac equation [54]. This can be done by assuming that the Coulomb potential energy involved and the kinetic energy of the electron are small in comparison to the resting energy of the particle. This derivation procedure had been shown in a number of textbooks and there is no need to repeat it here [54].

In summary, it can be demonstrated that our proposed basic wave equation is consistent with the commonly used quantum mechanical wave equations. Thus, there is no conceptual contradiction between our proposed wave model and the quantum equations used today. The only new suggestion in our model is that, the wave function in the quantum wave equation may have a more rich meaning than the traditional Copenhagen interpretation. That is, the wave function not only can give the probability of finding the particle, in addition to that, the wave function may also represent a physical oscillation of the vacuum medium, just analogous to the case of a photon.

\subsection{Is the Hypothesis of a Non-Empty Vacuum Consistent with Other Physical Theories? Is There Any Supporting Evidence?}

A key proposal in this work is that the vacuum is not an empty space. The idea of a non-empty vacuum had been considered previously by many well-known physicists, including Poincare, Planck, de Broglie, Schrödinger and Dirac. Many of them were aware that particles could be excitation waves of the vacuum. In their view, the vacuum cannot be empty.

In many branches of modern physics, including electrodynamics, atomic 
physics, quantum field theory and cosmology, the vacuum cannot be regarded as an empty space. In quantum electrodynamics, every oscillation mode is supposed to have a zero-point energy. Such energy is treated as a part of the vacuum system. In the Standard Model of cosmology today, it is assumed that the energy of our universe arises from quantum fluctuations in the vacuum [11] [12] [55]; the vacuum cannot be regarded as an empty space. Furthermore, the vacuum is also not considered empty in the current theories of particle physics. The vacuum is always regarded as the ground state. The physical fields are just excitations above the vacuum [13]. In the string theory, the vacuum is very complicated; it can be modeled as a web of strings [56] [57]. So, in most physical theories today, the vacuum is not regarded as an empty space.

\subsubsection{Experimental Evidence Indicating That the Vacuum Is Not Empty}

Furthermore, the idea of a non-empty vacuum has been tested in experiments. For example, it was demonstrated in the famous experiment of Lamb shift [58]. In the Dirac theory, the energy levels of ${ }^{2} S_{1 / 2}$ and ${ }^{2} P_{1 / 2}$ of a hydrogen atom are degenerate [59]. There should be no energy shift between them. But if the vacuum is not empty, the vacuum energy fluctuations can interact with the electrons in different orbitals. It would cause a very small energy shift. Such a shift was detected by Lamb and Retherford in 1947 [58]. In fact, the experimental value of this "Lamb shift" agreed well with the calculation of $H$. A. Bethe, who applied Kramers' idea of mass renormalization to account for the interactions between a free electron and the radiation field [59]. The Lamb shift has since played a significant role in demonstrating the importance of including vacuum energy fluctuations in theoretical calculations.

In addition to Lamb shift, non-empty properties of vacuum were also demonstrated in several other types of experiments, including spontaneous emission, vacuum polarization and the Casimir effect [60] [61] [62]. At present, people can no longer treat the vacuum as emptiness, although its physical properties are still under active investigation [50] [63] [64].

\subsubsection{Has the Michelson-Morley Experiment Already Proven That the Vacuum Is Empty?}

The idea that the vacuum behaves as a wave medium was well developed before the $20^{\text {th }}$ century; it was called the "Aether hypothesis". Many great physicists had believed in such a theory and made contribution to it [32]. It was only after the publications of the Michelson-Morley experiment and STR that this theory became disfavored. But the issue of whether the vacuum is an empty space or not was never clearly resolved.

In some textbooks, it was implied that the Michelson-Morley experiment had proven that the vacuum is empty. But this is really not true. Results of the optical interferometer experiment only showed that the transmission of electro-magnetic wave is the same in all inertial frames. This can be explained by requiring the wave equation of electro-magnetic radiation to be Lorentz invariant [13]. From 
the Maxwell's theory of light propagation, this is indeed the case.

However, there is a clear difference between the requirement of Lorentz invariance and the assumption of an empty vacuum. The latter is far more stringent; it assumes that the vacuum has no physical property, and, there is no fixed resting frame in our universe. In such a case, any inertial frame can be regarded as a stationary frame. In the case of Lorentz invariance, it only requires that a transformation of coordinate from $(t, x, y, z)$ into $\left(t^{\prime}, x^{\prime}, y^{\prime}, z^{\prime}\right)$ would not change the mathematical form of the equation of motion. It is purely a mathematical concept; it does not exclude the possibility that the universe may have a fixed resting frame. Whether or not the universe has a resting frame is a physical question which can only be settled in experiment.

One may say that, in addition to the Michelson-Morley experiment, there are also other reasons for rejecting the aether hypothesis. We would like to point out that, the wave model presented in this paper is not a revival of the previous aether hypothesis. As shown in Subsection 3.2, there is no contradiction between our wave model and the Michelson-Morley experiment. Furthermore, the vacuum in our model is not the same as the aether proposed in the $19^{\text {th }}$ century. First, the aether was supposed to be a medium filling only the space between matters; the vacuum in our model is a pre-existing entity that fills all space in our universe. Second, the aether was a hypothetical medium for transmitting electro-magnetic radiation only; the vacuum discussed here is a medium for all excitation waves, including both radiation waves and matter waves. In another word, not only photons are excitation waves of the vacuum, particles making up matters (such as electrons) are also excitation waves of the same vacuum. If one carefully examines the properties of the vacuum proposed in the wave model, one will find that all previous objections to the aether hypothesis would not apply to our vacuum hypothesis.

Furthermore, despite the common belief that the aether hypothesis was already abandoned, this is not really true. The existence of aether is still an open question in the minds of many great physicists. For example, although Einstein was initially against the aether hypothesis, he changed his mind later. When Einstein wrote his 1905 STR paper, he was not in favor of the aether hypothesis [5]. However, in 1920, when Einstein gave a talk entitled "Ether and the theory of relativity" at the Leiden University, he gave a very different view: "To deny the ether is ultimately to assume that empty space has no physical qualities whatever. The fundamental facts of mechanics do not harmonize with this view .... Recapitulating, we may say that according to the general theory of relativity space is endowed with physical qualities; in this sense, therefore, there exists an ether. According to the general theory of relativity space without ether is unthinkable; for in such space there not only would be no propagation of light, but also no possibility of existence for standards of space and time" [65].

P. A. M. Dirac was a pioneer who developed the relativistic electron quantum theory in 1928. In 1951, he published a letter in Nature entitled "Is there an 
aether?" He said: "Physical knowledge has advanced very much since 1905, notably by the arrival of quantum mechanics, and the situation has again changed. If one reexamines the question in the light of present-day knowledge, one finds that the aether is no longer ruled out by relativity, and good reasons can now be advanced for postulating an aether." [66]. Apparently, Dirac did not think the aether hypothesis is dead.

\subsection{Our Proposed Wave Model Can Be Tested in Experiment}

Our hypothesis that particles are excitation waves of the vacuum can be tested by experiment. If the vacuum is not empty, it will form a resting frame in our universe, and the rest mass of a particle can only be defined relative to this resting frame. In the Michelson-Morley experiment [16], it only showed that there is no resting frame for a photon. But, since the photon has no rest mass and its speed always equals to $c$, one cannot measure its mass in different inertial frames. In order to test our hypothesis, one needs to conduct an interference experiment using particles with nonzero rest mass.

Recently, we suggested that one can test whether there is a resting frame in our universe by precisely measuring the moving mass of two particles traveling in opposite directions [67]. We know the moving mass of a particle is speeddependent. If there is a resting frame in our universe, our laboratory on Earth will be in motion relative to this frame. Then, one will observe a difference in the moving mass when the particle moves in opposite directions. This is because the total speed of the particle will depend on both the particle velocity relative to the laboratory frame and the velocity of the laboratory frame relative to the vacuum.

We proposed to conduct this experiment in a way similar to the MichelsonMorley experiment. That is, we can take advantage of the fact that the experimental apparatus is fixed at the surface of the Earth; its orientation depends on the Earth's rotation. We can change the direction of the particle movement by simply repeating the experiment in different times of the day or in different seasons of the year. Based on the known speed of the Earth (taking into consideration the Earth's orbital speed, the speed of the Sun in our galaxy, and the speed of the Milky Way vs the rest of the universe), we estimated that the amplitude of the mass variation due to the change of apparatus orientation would be in the order of $2 \times 10^{-4}$ [67]. Such a frame-dependence effect on the particle mass should be measurable using existing technology.

\section{Conclusions}

1) We have conducted a detailed literature review on the derivation of two important relations about particle mass, i.e., the speed-dependence of mass and the mass-energy equivalence relation. We found that, contrary to common belief, these relations were not derived based on the first postulate of Einstein's theory of relativity. Instead, their derivations were more based on Einstein's intuitive thinking that radiation and matters behave similarly. 
2) Based on this hint, we explored if the non-Newtonian properties of mass can be explained on the physical basis of wave-particle duality. We know both photon and electron can behave as a particle as well as a wave. Can such a wave nature account for the deviations from Newtonian mechanics?

3) We developed a wave model to describe the movement of matter wave representing a free particle. Our basic assumption is that all particles (with or without rest mass) are excitation waves of the vacuum. Using this model, the relations $E=m c^{2}$ and $m=m_{0}\left(1-v^{2} / c^{2}\right)^{-1 / 2}$ can be directly derived. This demonstrated that both the speed-dependence of mass and the mass-energy equivalence can be based on the wave properties of a particle.

4) This wave model predicts that light must travel at a constant speed $c$ and no particle can travel faster than the speed of light.

5) Furthermore, this wave model explains why particles can be created in the vacuum.

6) A potential application of this wave model is to explain the possible origin of dark matter. Based on astronomical observations, we know galaxies contain a large amount of dark matter in addition to visible matter. At this point, no particle has been identified as the source of dark matter. From our wave model, one can identify dark matter as excitation waves of the vacuum that are uncharged and do not involve in strong interaction. Since these excitation waves contain energy, they will have equivalent mass and thus can naturally exhibit a gravitational effect.

\section{Acknowledgements}

I would like to thank Profs. J. A. Wheeler and H. E. Rorschach for their encouragement at the early stage of this work. I also thank Drs., Bambi Hu, Don Tow, Zhaoqing Zhang and Xiangrong Wang for their suggestions and comments. I thank Ms. Lan Fu for her assistance. This work was partially supported by the Research Grant Council of Hong Kong (RGC 660207) and the Macro-Science Program, Hong Kong University of Science and Technology (DCC 00/01.SC01).

\section{References}

[1] Cockcroft, J.D. and Walton, E.T.S. (1932) Proceedings of the Royal Society of London $A, 137,229-242$. https://doi.org/10.1098/rspa.1932.0133

[2] Bainbridge, K.T. (1933) Physical Review, 44, 123. https://doi.org/10.1103/PhysRev.44.123.2

[3] Greene, G.L., Dewey, M.S., Kessler, E.G. and Fischbach, E. (1991) Physical Review $D$, 44, R2216. https://doi.org/10.1103/PhysRevD.44.R2216

[4] Rainville, S., et al. (2005) Nature, 438, 1096-1097. https://doi.org/10.1038/4381096a

[5] Einstein, A. (1905) Annalen der Physik, 322, 891-921. https://doi.org/10.1002/andp.19053221004

[6] Coughlan, G.D. and Dodd, J.E. (1991) The Ideas of Particle Physics: An Introduction for Scientists. Cambridge University Press, Cambridge.

[7] Thomson, J.J. (1881) Philosophical Magazine, 11, 229-249. 
https://doi.org/10.1080/14786448108627008

[8] Heaviside, O. (1889) Philosophical Magazine, 27, 324-339. https://doi.org/10.1080/14786448908628362

[9] Poincaré, H. (1900) Archives Néerlandaises des Sciences Exactes et Naturelles, 2, 252-278.

[10] Lorentz, H.A. (1904) Proceedings of the Royal Netherlands Academy of Arts and Science, 6, 809-831.

[11] Weinberg, S. (2008) Cosmology. Oxford University Press, Oxford.

[12] Guth, A.H. and Kaiser, D.I. (2005) Science, 307, 884-890. https://doi.org/10.1126/science.1107483

[13] Ryder, L.H. (1996) Quantum Field Theory. Cambridge University Press, Cambridge. https://doi.org/10.1017/CBO9780511813900

[14] Chang, D.C. (2017) Chinese Physics B, 26, Article ID: 040301.

[15] Fadner, W.L. (1988) American Journal of Physics, 56, 114-122. https://doi.org/10.1119/1.15713

[16] Michelson, A.A. and Morley, E.W. (1887) American Journal of Science, 34, 333-345. https://doi.org/10.2475/ajs.s3-34.203.333

[17] Kaufmann, W. (1902) Physikalische Zeitschrift, 4, 54-57.

[18] Bucherer, A.H. (1909) Annalen der Physik, 333, 513-536. https://doi.org/10.1002/andp.19093330305

[19] French, A.P. (1968) Special Relativity. Norton, 1-29.

[20] Rothman, T. (2015) Was Einstein the First to Invent $E=m c^{2}$ ? https://www.scientificamerican.com/article/was-einstein-the-first-to-invent-e-mc2/

[21] Wien, W. (1901) Annalen der Physik, 310, 501. https://doi.org/10.1002/andp.19013100703

[22] Abraham, M. (1902) Physikalische Zeitschrift, 4, 57-63.

[23] Hasenöhrl, F. (1904) Annalen der Physik, 15, 344-370. https://doi.org/10.1002/andp.19043201206

[24] Hasenöhrl, F. (1905) Annalen der Physik, 16, 589. https://doi.org/10.1002/andp.19053210312

[25] Einstein, A. (1905) Annalen der Physik, 323, 639-641. https://doi.org/10.1002/andp.19053231314

[26] Ives, H.E. (1952) Journal of the Optical Society of America, 42, 540-543. https://doi.org/10.1364/JOSA.42.000540

[27] Einstein, A. (1906) Annalen der Physik, 325, 627-633. https://doi.org/10.1002/andp.19063250814

[28] Einstein, A. (1907) On the Inertia of Energy Required by the Relativity Principle. In: Stachel, J., et al., Eds., Beck, A., Trans., The Collected Papers of Albert Einstein, the Swiss Years: Writings, 1900-1909, Vol. 2, Princeton University Press, Princeton, 238-250.

[29] Hecht, E. (2011) American Journal of Physics, 79, 591-600. https://doi.org/10.1119/1.3549223

[30] Jammer, M. (2000) Concepts of Mass in Contemporary Physics and Philosophy. Princeton University Press, Princeton, 62-89. https://doi.org/10.1063/1.1341924

[31] Ohanian, H.C. (2012) American Journal of Physics, 80, 1067-1071. https://doi.org/10.1119/1.4748812 
[32] Whittaker, E. (1951) A History of the Theories of Aether and Electricity. Nelson, London.

[33] Arzelies, H. (1966) Rayonnement et Dynamique du Corpuscule Charge Fortement Accelere. Gauthier-Villars, Pairs, 74-79.

[34] Stachel, J. and Torretti, R. (1982) American Journal of Physics, 50, 760-763. https://doi.org/10.1119/1.12764

[35] Hecht, E. (2009) American Journal of Physics, 77, 799-806. https://doi.org/10.1119/1.3160671

[36] Rogers, M.M., McReynolds, A.W. and Rogers, F.T. (1940) Physical Review, 57, 379-383. https://doi.org/10.1103/PhysRev.57.379

[37] Einstein, A. (1940) Science, 91, 487-492. https://doi.org/10.1126/science.91.2369.487

[38] Einstein, A. (1946) Technion Yearbook, 5, 16-17.

[39] Einstein, A. (1905) Annalen der Physik, 17, 132-148. https://doi.org/10.1002/andp.19053220607

[40] Millikan, R.A. (1914) Physical Review, 4, 73.

[41] Compton, A.H. (1923) Physical Review, 21, 483-502. https://doi.org/10.1103/PhysRev.21.483

[42] Thomson, G.P. and Reid, A. (1927) Nature, 119, 890. https://doi.org/10.1038/119890a0

[43] Davisson, C.J. and Germer, L.H. (1927) Nature, 119, 558-560. https://doi.org/10.1038/119558a0

[44] Planck, M. (1900) Verhandlungen der Deutschen Physikalischen Gesellschaft, 2, 237.

[45] Knoll, M. and Ruska, E. (1932) Zeitschrift für Physik, 78, 318-339. https://doi.org/10.1007/BF01342199

[46] Messiah, A. (1965) Quantum Mechanics, Vol. 1. Wiley, New York, 45-74.

[47] Chang, D.C. (2017) On the Wave Nature of Matter: A Transition from Classical Mechanics to Quantum Mechanics. arXiv:physics/0505010 [physics.gen-ph]

[48] Bach, R., et al. (2013) New Journal of Physics, 15, Article ID: 033018. https://doi.org/10.1088/1367-2630/15/3/033018

[49] Maxwell, J.C. (1865) Philosophical Transactions of the Royal Society of London, 155, 459-512. https://doi.org/10.1098/rstl.1865.0008

[50] Chang, D.C. and Lee, Y. (2015) Journal of Modern Physics, 6, 1058-1070. https://doi.org/10.4236/jmp.2015.68110

[51] Chang, D.C. (2017) What Is the Physical Meaning of Mass in View of Wave-Particle Duality? A Proposed Model. arXiv:physics/0404044 [physics.gen-ph]

[52] Arfken, G.B. and Weber, H.J. (1995) Mathematical Methods for Physicists. 2nd Edition, Cambridge University Press, Cambridge.

[53] Dirac, P.A.M. (1981) The Principles of Quantum Mechanics. Clarendon Press, Oxford, 253-275.

[54] Cottingham, W.N. and Greenwood, D.A. (1998) An Introduction to the Standard Model of Particle Physics. Cambridge University Press, Cambridge, 71-72.

[55] Steinhardt, P.J. and Turok, N. (2002) Physical Review D, 65, Article ID: 126003. https://doi.org/10.1103/PhysRevD.65.126003 
[56] Candelas, P., et al. (1985) Nuclear Physics B, 258, 46-74. https://doi.org/10.1016/0550-3213(85)90602-9

[57] Wen, X. (2003) Physical Review D, 68, Article ID: 065003. https://doi.org/10.1103/PhysRevD.68.065003

[58] Lamb, W.E. and Retherford, R.C. (1947) Physical Review, 72, 241. https://doi.org/10.1103/PhysRev.72.241

[59] Bethe, H.A. (1947) Physical Review, 72, 339. https://doi.org/10.1103/PhysRev.72.339

[60] Goy, P., Raimond, J.M., Gross, M. and Haroche, S. (1983) Physical Review Letters, 50, 1903. https://doi.org/10.1103/PhysRevLett.50.1903

[61] Levine, I., et al. (TOPAZ Collaboration) (1997) Physical Review Letters, 78, 424-427. https://doi.org/10.1103/PhysRevLett.78.424

[62] D’Hoker, E. and Sikivie, P. (1993) Physical Review Letters, 71, 1136-1139. https://doi.org/10.1103/PhysRevLett.71.1136

[63] Boi, L. (2011) The Quantum Vacuum. Johns Hopkins University Press, Baltimore.

[64] Oi, D.K.L., Potoček, V. and Jeffers, J. (2013) Physical Review Letters, 110, Article ID: 210504. https://doi.org/10.1103/PhysRevLett.110.210504

[65] Einstein, A. (1920) Ether and the Theory of Relativity (Speech at University of Leiden, May 5, 1920). In: Janssen, M., et al., Eds., Engel, A., Trans., The Collected Papers of Albert Einstein, the Swiss Years: Writings, 1918-1921, Vol. 7, Princeton University Press, Princeton, 160-182.

[66] Dirac, P.A.M. (1951) Nature, 168, 906-907. https://doi.org/10.1038/168906a0

[67] Chang, D.C. (2017) The European Physical Journal Plus, 132, 140. https://doi.org/10.1140/epjp/i2017-11402-4

[68] Kaufmann, W. (1901) Göttinger Nachrichten, 2, 143-168.

[69] Einstein, A. (1906) The Principle of Conservation of Motion of the Center of Gravity and the Inertia of Energy. In: Stachel, J., et al., Eds., Beck, A., Trans., The Collected Papers of Albert Einstein, the Swiss Years: Writings, 1900-1909, Vol. 2, Princeton University Press, Princeton, 200-206.

[70] Einstein, A. (1907) On the Relativity Principle and the Conclusions Drawn from It. In: Stachel, J., et al., Eds., Beck, A., Trans., The Collected Papers of Albert Einstein, the Swiss Years: Writings, 1900-1909, Vol. 2, Princeton University Press, Princeton, 252-311.

[71] Planck, M. (1908) Annalen der Physik, 331, 1. https://doi.org/10.1002/andp.19083310602

[72] Einstein, A. (1909) On the Development of Our Views Concerning the nature and Constitution of Radiation. In: Stachel, J., et al., Eds., Beck, A., Trans., The Collected Papers of Albert Einstein, the Swiss Years: Writings, 1900-1909, Vol. 2, Princeton University Press, Princeton, 379-394.

[73] Einstein, A. (1911) On the Influence of Gravitation on the Propagation of Light. In: Klein, M.J., et al., Eds., Beck, A., Trans., The Collected Papers of Albert Einstein, the Swiss Years: Writings, 1909-1911, Vol. 3, Princeton University Press, Princeton, 379-387.

[74] Einstein, A. (1912) Manuscript on the Special Theory of Relativity, Section 14, The Inertia of Energy. In: Klein, M.J., et al., Eds., Beck, A., Trans., The Collected Papers of Albert Einstein, the Swiss Years: Writings, 1912-1914, Vol. 4, Princeton University Press, Princeton, 50-54. 
[75] Einstein, A. (1912-1914) Manuscript on the Special Theory of Relativity. In: Klein, M.J., et al., Eds., Beck, A., Trans., The Collected Papers of Albert Einstein, the Swiss Years: Writings, 1912-1914, Vol. 4, Princeton University Press, Princeton, 3-88.

[76] Einstein, A. (1922) The Meaning of Relativity. Princeton University Press, Princeton. https://doi.org/10.4324/9780203449530

[77] Einstein, A. (1935) Bulletin of the American Mathematical Society, 41, 223-230. https://doi.org/10.1090/S0002-9904-1935-06046-X 


\section{Appendix A: Historical Review on the Derivation of the Mass-Energy Equivalence Relation}

The following is a list of major publications contributing to the derivation of the mass-energy equivalence relation, particularly the works of Einstein. This list was compiled mainly based on the reviews of Rothman, Fadner and Hecht [15] [20] [29].

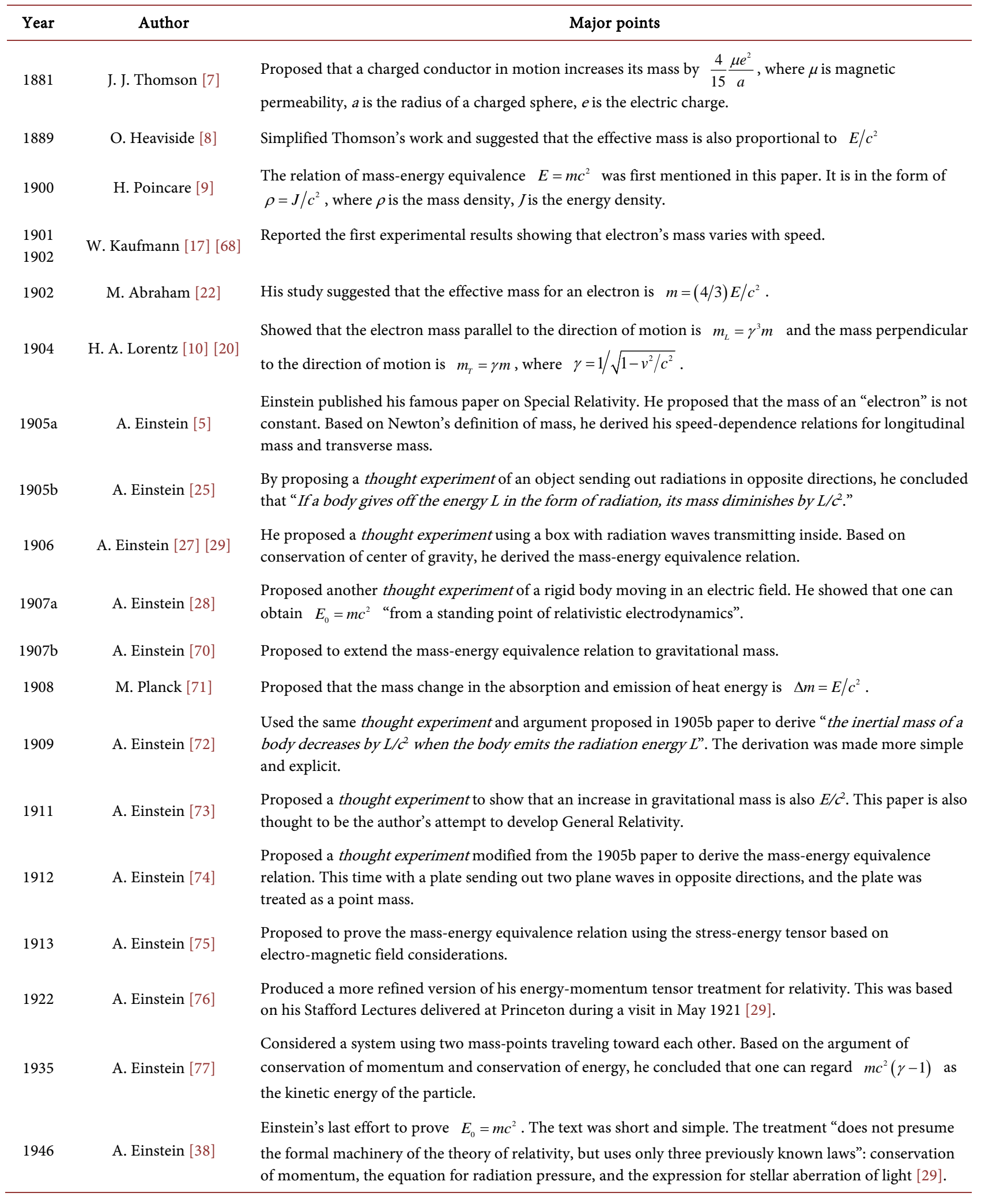

